\title{
CILIARY PROPULSION OF OBJECTS IN TUBES: WALL DRAG ON SWIMMING TETRAHYMENA (CILIATA) IN THE PRESENCE OF MUCIN AND OTHER LONG-CHAIN POLYMERS
}

\author{
Bx H. WINET \\ Division of Engineering and Applied Science, California Institute of Technology, \\ Pasadena, California 9I I 25
}

(Received 4 fuly 1975)

\section{SUMMARY}

The lubrication effect of three long-chain polymers - mucin, methylcellulose and Ficoll - on ciliary propulsion in tubes is measured by plotting the relative velocities of swimming ciliates as a function of tube bore diameter. Mucin shows the most unequivocal lubrication, which is found at concentrations between $\circ \%$ and $9 \cdot 1 \%(\mathrm{w} / \mathrm{v})$. This observation, coupled with viscometric measurements which show that ciliary tip shear rates are sufficient to solate mucin, serve as the groundwork for a model of mucin lubrication which explains the optimized lubrication behaviour of thixotropic gelating polymers as an expression of the responses to shear by the various stages of polymer clustering during the gelation process. In addition to the lubricative effect, another wall drag reduction effect by mucin was measured in the clearance region beyond the lubrication layer. This apparent viscosity reduction is optimized in the concentration range between $\mathrm{I} \cdot 7$ and $4.1 \%$ mucin and may also be explained in terms of the properties of gel clustering.

\section{INTRODUCTION}

Cilia in multicellular systems (and apparently some unicellular systems (Winet \& Jones, 1975)) operate predominately in mucus or other suspensions of long-chain polymers, and are often bounded by walls or propelling a large inert solid (e.g. an ovum), so the propulsion system must be ultimately analysed as ciliary propulsion of/in a non-Newtonian fluid near a wall. It appears that no rigorous attempt has been made to model mathematically such a propulsion system since the simple analysis of Barton \& Raynor $(1967)$. Some theoreticians have since treated ciliary propulsion in mucus (Blake, 1973a, $b$; Ross, 1971; Miller, 1969) and some ciliary propulsion in tubes (Blake, I973c; Lardner \& Shack, 1972).

The system of interest here consists of a wall (with or without cilia), an object (which may or may not bear cilia) for the cilia to propel, and a non-Newtonian medium in which the propelled object must travel: an example is the ciliated oviduct-ovum system. Characterization of such a three-component system, in such a way as to generate theoretical relations readily testable by observation, is still remote - primarily because the fluid mechanics of non-Newtonian rheology are too complex for classical 
continuum approaches (Walters, 1973). Accordingly, the state of the art is such than we cannot even draw a dependable theoretical flow-velocity profile for this system which could be compared with the profile for the Newtonian fluid system (see Winet, I973).

Experimentalists can facilitate the development of a simple mechanical model by showing where reasonable approximations can be made in the system; for example, where steady-state kinematics may be assumed, where the fluid can be approximated as being Newtonian and where the wall effect begins. At present we cannot obtain this information with any consistency from ciliated surfaces in vivo, primarily because of the wide variation in the condition of the mucus of a given tissue even in the same individual (Eliezer, 1974) and the possible disruption of normal ciliary activity in an excized sample. The experiments are more tractable, however, if we replace in vivo mucus with a standardized preparation of the main constituent of mucus, the glycoprotein mucin, and the ciliated epithelium with a ciliated micro-organism. While it is true that the transformation from a fixed to a moving ciliated surface changes the flow-velocity profile, the differences are relatively small (we are assuming here, of course, that the three-component-system model will still conform to a quasi-steady form of the hydrodynamic equations of motion) and readily measured in a near field (the region close to the ciliated surface) analysis of the type conducted by Cheung \& Winet (1974) for Spirostomum.

In the present report we shall be concerned with the general effects of mucin, and two other long-chain polymers of biological interest, for purposes of comparison, on ciliary propulsion in rigid tubes with particular emphasis on one of the usually assumed functions of mucus in tubes - lubrication (Marshall, 1972). This work is an extension of a previous report which established the control system of ciliary propulsion in tubes containing only the normal medium of the micro-organism (Winet, 1973).

\section{MATERIALS AND METHODS}

The ciliated micro-organism utilized for these observations was the protozoan Tetrahymena, which was made to swim in suspensions of polymer in tubes of various bore diameters. Observations were made at two precision levels. All three polymers were evaluated utilizing the methods described previously (Winet, I973). A more exact measurement of the behaviour of each organism was carried out, however, for the mucin system. By fitting a Zeiss dry darkfield condenser (numerical aperture 0.70 ) to a Nikon LKe microscope and utilizing Nikon objectives with smaller numerical apertures, a photographable darkfield image of the organisms could be obtained in the oil-immersed capillary tube. The images were photographed on $35 \mathrm{~mm}$ film, Kodak 2475, utilizing Chadwick-Helmuth Strobex 360 xenon-arc illumination. Possible UV effects on the cilia were reduced by filtering the light with a $\mathrm{K}_{2}$ (Kodak) filter. The resulting negatives were analysed on a DASA 330 Reader-Printer.

Suspensions including the ciliate were prepared as follows.

Mucin. Dropper pipettes were calibrated to determine volume per drop and then used to add samples from 2-week-old test-tube axenic cultures of Tetrahymena growing in $2 \%$ proteose-peptone to pre-weighed samples of dehydrated bovine submaxillary mucin, BSM (Sigma), in a depression slide. The resulting suspension, 
ranging from $5 \cdot 16 \%(\mathrm{w} / \mathrm{v})$ down, was stirred with a toothpick until it appeared homogeneous. The stirring was repeated each time a sample was removed for observation. No preparation was kept beyond $9 \mathrm{~h}$ and all observations were conducted at $20^{\circ} \mathrm{C}$.

Methylcellulose. This polysaccharide is widely used to measure energy expended by ciliary and flagellar contraction against external viscosity. A powder rated at $15 \mathrm{cP}$ (Fisher Chemical) was used. A stock $5.56 \%$ (w/v) suspension was prepared by mixing, heating and diluting an $11 \cdot 12 \%(\mathrm{w} / \mathrm{v})$ suspension with distilled $\mathrm{H}_{2} \mathrm{O}$. The stock suspension was allowed to remain at $20^{\circ} \mathrm{C}$ for I week prior to its use. It retained its homogeneous appearance without refrigeration throughout the period of storage and use. When used, the necessary volumes of stock suspension were mixed with appropriate volumes of culture, and stirred as with the mucin preparations.

Ficoll 400 is a copolymer of sucrose and epichlorohydrin, with the attractive property of low tonicity at relatively high concentrations. This polymer is considered a possible replacement for methylcellulose in studies of ciliary and flagellar locomotion. Because it dissolves easily and is readily obtainable in large quantities, solutions made by dissolving Ficoll in appropriate volumes of culture were prepared as needed and remained homogeneous without stirring. The concentrations ranged from $25 \%(w / v)$ down.

In all cases the organisms were allowed to equilibrate with their test suspensions for at least $\mathrm{I} h$ before observations were made.

Measurements of apparent viscosity, $\eta$, were carried out with the aid of a Brookfield LVF rotating-spindle viscometer. Data were obtained with the no. 2 spindle (diameter $\mathrm{r} \cdot 87 \mathrm{~cm}$ ) rotating such that the shear rates fell into the range $\mathrm{I}^{\cdot} 5^{-1} 5^{-2} \mathrm{~s}^{-1}$. For these experiments cultures were replaced by appropriate volumes of sterile $2 \%$ proteosepeptone, and BSM was replaced by a more crude extract of porcine gastric mucin, PGM (Sigma). The concentration of PGM used for these observations was higher than corresponding BSM concentrations because PGM has a lower sialic acid content ( $\mathrm{r} \%$ as compared with $5 \%$ ) and the sialic acid content of mucin is a major determinant of its viscous characteristics. The substitution of PGM for BSM was made on purely economic grounds.

The velocity observations were related to viscosity measurements as follows. First, three types of velocity were distinguished. The base-line control velocity, $U_{0}$, was the swimming speed of a ciliate in its normal culture medium in a tube so wide that the organism could be considered in an 'unbounded' system. This velocity is equivalent to the control for a previous investigation (Winet, 1973). The polymer control velocity, $U_{\infty}$, was obtained from organisms swimming in polymer suspension in unbounded systems. The test velocity, $U$, was a measure of swimming in polymer suspensions in tubes of bore diameter smaller than for the $U_{\infty}$ case. By relating $U_{\infty} / U_{0}$ and $U / U_{\infty}$ to polymer concentration, and the latter to viscosity, a measure of velocity as a function of apparent viscosity of a given polymer suspension could be obtained (presuming a reproducible if not small error from the use of PGM in place of BSM). The relative values of $U / U_{\infty}$ as a function of clearance - the distance between body surface and tube wall - provide the coordinates for the desired velocity profile.

In the non-photographic experiments there were two measurement populations for which averages were obtained: a size population which was assumed to be a random sampling from each suspension of polymer-plus-culture, and a velocity population 
which included only those organisms swimming continuously over a marked distance. Accordingly, in all plots of clearance $v$. velocity both vertical and horizontal variance bars appear and are based upon the formula for the sample standard deviation, $s$, where

$$
s=\sqrt{(\mathrm{I} / n-\mathrm{I}) \Sigma\left(x_{1}-\bar{x}\right)^{2} .}
$$

In the case where an axis represented combined population data (e.g. $U / U_{\infty}$ is calculated with data from the $U$ and the $U_{\infty}$ populations) an $s^{\prime}$ value for standard deviation was used where

$$
\left.s^{\prime}=s_{a} \frac{\partial F}{\partial a}+s_{b} \frac{\partial F}{\partial b} \dagger \quad \text { (Bevington, } 1969\right) \text {. }
$$

Here $F=F(a, b)$ and $s_{a}$ and $s_{b}$ are the $s$ values for the $a$ and $b$ populations (e.g. $\left.F\left(U, U_{\infty}\right)=U / U_{\infty}\right)$.

In the photographic experiments the $s^{\prime}$ value was determined by comparing the measured $U$ values with a stopwatch-determined $U_{\infty}$. A separate $U_{\infty}$ was determined (all sample sizes were ${ }_{15}$ ) during each photographic series (i.e. for each preparation).

In order to obtain some indication of what may be expected from an investigation of suspension flow in the near field - in and close to the ciliary layer - an estimate of the local viscosity at the cilia, $\eta_{c}$, is also obtained. This estimate is necessary because, for non-Newtonian fluids, values of $\eta$ obtained at shear rates far removed from their in vivo values lead to a false picture of the stresses on cilia beating in a long-chain polymer suspension. By utilizing published data and measurements obtained by viscometry, a power law estimate of $\eta_{c}$ is readily calculated. The resulting values for $\eta_{c}$ could then be used in place of $\eta$ in the model. The model at this point would be improved but still not accurate because not only is it erroneous to assume $\eta=\eta_{c}$, but in a more subtle way it is incorrect to assume that $\eta_{c}$ at the ciliary tip is a true measure of $\eta_{c}$ over an entire cilium or ciliated surface. A non-Newtonian fluid reacts at every element of a cilium in accordance mainly with the shear generated in the neighbourhood of that element and the true $\eta_{c}$ reflects the effect of all the elements. Brokaw (1966) emphasized the problem of eliminating this final error, but the approximate nature of the present study does not require a full accounting of the true $\eta_{c}$.

\section{RESULTS}

The increase in absolute $\eta$ with increase in concentration, for a given shear rate, is well documented for mucin (Gibbons \& Glover, 1959; Tsuiki, Hashimoto \& Pigman, 1961), methylcellulose (Machemer, 1972) and Ficoll (Pharmacia, 1968), and is confirmed by the measurements presented in Fig. I. The non-linearity of this relationship is evident in Fig. I. A predictable corollary to this relationship is the non-linear decrease in propulsion velocity $\left(U_{\infty} / U_{0}\right)$ with increase in polymer concentrations, as shown in Fig. 2. This result is in qualitative agreement with the reported effects of methylcellulose concentration on ciliary beat frequency (Machemer, 1972; Preston, 1972).

$\dagger$ The use of this formula is based upon the assumption that variations in swim speed inherent in any sample from a viable Tetrahymena culture are a far greater contributor to sample standard deviations than experimental error is. The validity of this assumption requires that both $U$ and $U_{\infty}$ be determined from the same preparation, a condition which is accepted here to remain intact when data from two preparations are combined provided there is no significant difference between the two mean $U_{\infty}$. 


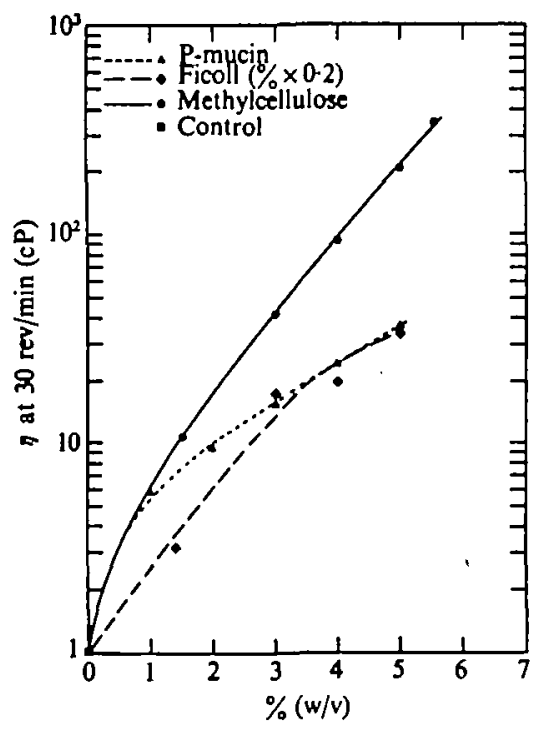

Fig. I

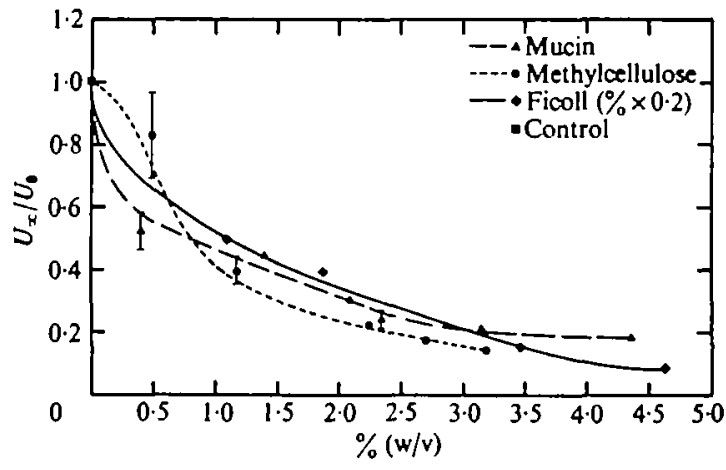

Fig. 2

Fig. I. Semi-log plot of apparent viscosity as a function of polymer suspension concentration for three long-chain polymers. The control is $2 \%$ proteose-peptone.

Fig. 2. The effect of polymer suspension concentration on the swim velocity of Tetrahymena in 'unbounded' medium. $U_{\infty}$ is the velocity in polymer suspension and $U_{0}$ is the velocity in normal culture medium. Variances are standard sample deviations. Values lacking variance bars had variance values too small to illustrate.

In the presence of a boundary, in this case a glass-tube wall, the non-Newtonian fluid complexities are further compounded, as indicated in the wall-drag velocity profiles for various concentrations of the three polymer suspensions which are presented in Figs. 3-5. In these plots the abscissa is the reduced velocity $U / U_{\infty}$ and the ordinate is the reduced clearance $C / r$, where $C$ is the distance between the non-ciliary cell surface and the nearest wall and $r$ is $7 \mu \mathrm{m}$, the average cilium length for Tetrahymena. One should, however, interpret these graphs with caution. Unlike the photographic experiments, it was not possible to measure clearance for a given organism and velocity simultaneously. This condition generated two sources of variance in the results. First, there was the normal variation (rarely over $12 \%$ ) in cell width for a random sample of culture cells. Secondly, there was some selection for the narrower organisms by the narrower tubes. Accordingly, one may expect that actual clearance may be higher than reported. A further source of clearance variation arises from the characteristics of the cilia. First, although a constant length of $r=7 \mu \mathrm{m}$ was utilized, these organelles do range from $6-8 \mu \mathrm{m}$. Secondly, as observed by Preston (1972), Tetrahymena cilia neither conform to a straight line shape nor do they ascend to a beat angle, $\theta$, which is normal to the body surface durring swimming. Indeed, viscous drag from both swimming and increased bulk fluid viscosity tends to bend the ciliary beat axis posteriad and convert the beat form into a 'helicoidal' shape (Machemer, 1972; Cheung \& Winet, 1974). Hence, if we assumed for the sake of argument that a fully extended cilium was bent back so that its tip was $\frac{1}{2}$ a ciliary length from the body surface, then its inclination need only approach to within $\sin ^{-1} 0.5=\theta=30^{\circ}$ of the 


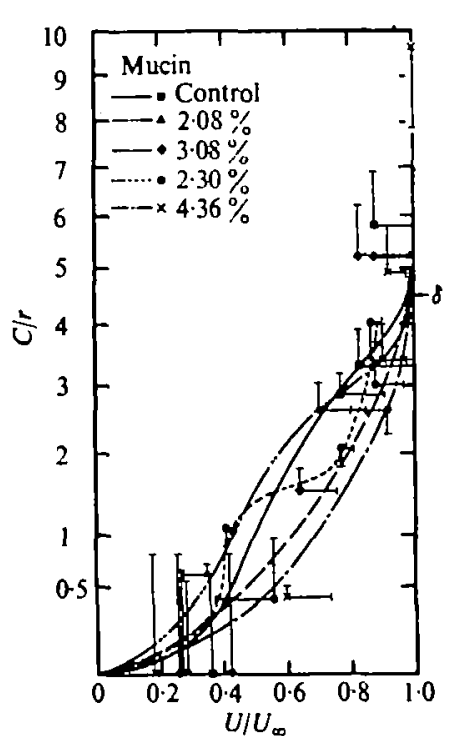

Fig. 3

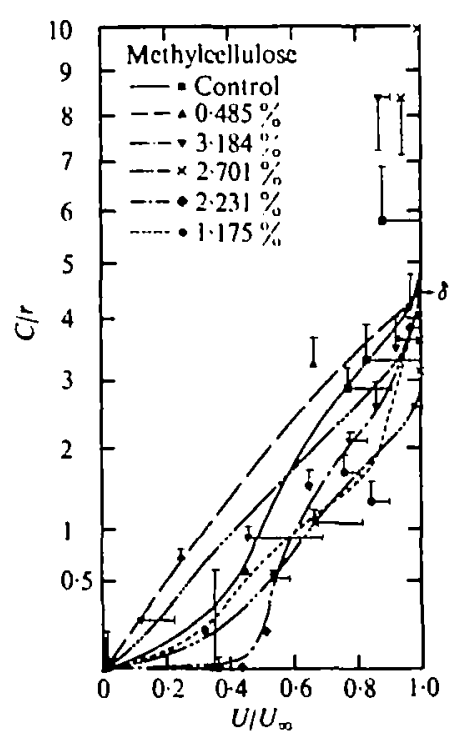

Fig. 4

Fig. 3. A two-thirds power plot of the effect of clearance in a tube of mucin suspension on the reduced swim velocity (a propulsion velocity profile). $U$ is the bounded medium velocity. Variances are standard sample deviations of measurements of organism width (vertical bars) and velocity (horizontal bars). Values at $C / r=0$ without variance bars are single values. Other values lacking variance bars had variance values too small to illustrate. The meaning of $\delta$ is explained in the text.

Fig. 4. A two-thirds power plot of the effect of clearance in a tube of methylcellulose suspension on the reduced swim velocity. Plot details as for Fig. 3.

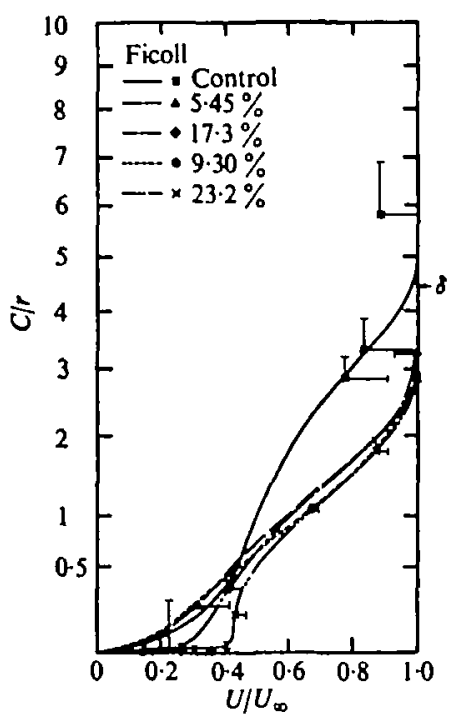

Fig. 5. A two-thirds power plot of the effect of clearance in a tube of Ficoll solution on the reduced swim velocity. Plot details as for Fig. 3 . 
body surface to allow the organism to fit into a tube with bore clearance $C / r=0.5$. Given a helicoidal beat axis, angle $\theta$, of $30^{\circ}$ and the less-affected cilia at the curved fore and aft portions of the cell, there is no reason to believe that such a tetrahymenid would be non-motile. These factors do, however, raise the question of the nature of the beat form for a tethered tetrahymenid and for ciliated epithelium. The effect of an increased bulk fluid viscosity on ciliary beat will surely be similar, and a flowing bulk fluid should in turn help bend the ciliary beat axis in the direction of propulsion. The feedback nature of this system will complicate the theoretical transformation from an affixed to a moving organism flow field which was referred to in the Introduction. (Although such a transformation will still be relatively simple if we make $\theta$ a constant for each flow velocity profile and then compare each affixed ciliary field profile with the profile of a swimming organism with the same $\theta$.)

The 'natural' polymers display no clear pattern in Figs. 3 and 4 although, in general, a lubrication tendency is detectable for $C / r$ values between $\mathrm{I}$ and 3 where the polymer concentration exceeds some minimum. For mucin this minimum is above $2.08 \%$ and for methylcellulose above $0.485 \%$. A more distinct and uniform lubrication pattern characterizes the Ficoll system where the relative lubrication effect is essentially independent of concentration. In all three investigations where lubrication is present, wall drag disappears at a smaller clearance value $\left(S_{v 0}\right)$ for the polymer system than for the control system (the control plots become asymptotic at some value about $4 r$ ). The photographic investigation data for mucin generate the plots of Figs. 6 and 7 and show a pattern which was masked by the multiplicity of data variance which accompanies the timed experiments. Fig. 6 shows each data set in detail and Fig. 7 compares the resulting plots for all mucin concentrations. The control data from these investigations follow a double-hump curve and form three distinct relationships with the mucin results which are delineated by three clearance ranges. In the range $0.45<\mathrm{C} / r$ $\leqslant \mathrm{r} \cdot \mathrm{O}$ all $U / U_{\infty}$ values are higher for the mucin systems indicating a lubrication effect at these clearances. Over the second range $\mathrm{r} \cdot 0<C / r<\mathrm{r} \cdot 5$ the control values share many coordinates with mucin system data as propulsion of the organism rapidly approaches $U_{\infty}$. The final range extends upward from $C / r=3.5$ to the various values at which wall drag disappears.

In this final range the behaviour of the mucin systems takes on a rather optimized character. An organism swimming in mucin concentrations within the range $1 \cdot 7 \%$ $<M<4.1 \%$ (see Fig. 7) swims nearer $U_{\infty}$ than is the case for the control system while one swimming at concentrations outside this range cannot approach $U_{\infty}$ as well as its counterpart in the control system. The wall effect in the control system appears to commence at $6 \cdot 5^{r}$ from the cell surface. This value compares favourably with the $6.24 r$ which can be calculated from the value obtained in an earlier work (Winet (1973) - here the value $S_{w}$ for Tetrahymena is given in body semi-axis lengths). In contrast, the wall effect in the $2.9 \%$ mucin system commences at $3.5 \mathrm{r}$ from the cell surface a value which is, curiously, close to the $\delta=3.42 r$ calculated from the $\delta$ value for Tetrahymena of the same work. It will be noted that the optimized velocity 'enhancement' characteristic of the $C / r \geqslant 3.5$ range is not referred to as 'lubrication', the effect to which this investigation was originally addressed. This change in approach arises from the consideration that the slenderness ratio of Tetrahymena is so small (rarely over $2: 1$ ) that at a distance of $3 \cdot 5 r$ non-lubricative hydrodynamic wall effects 

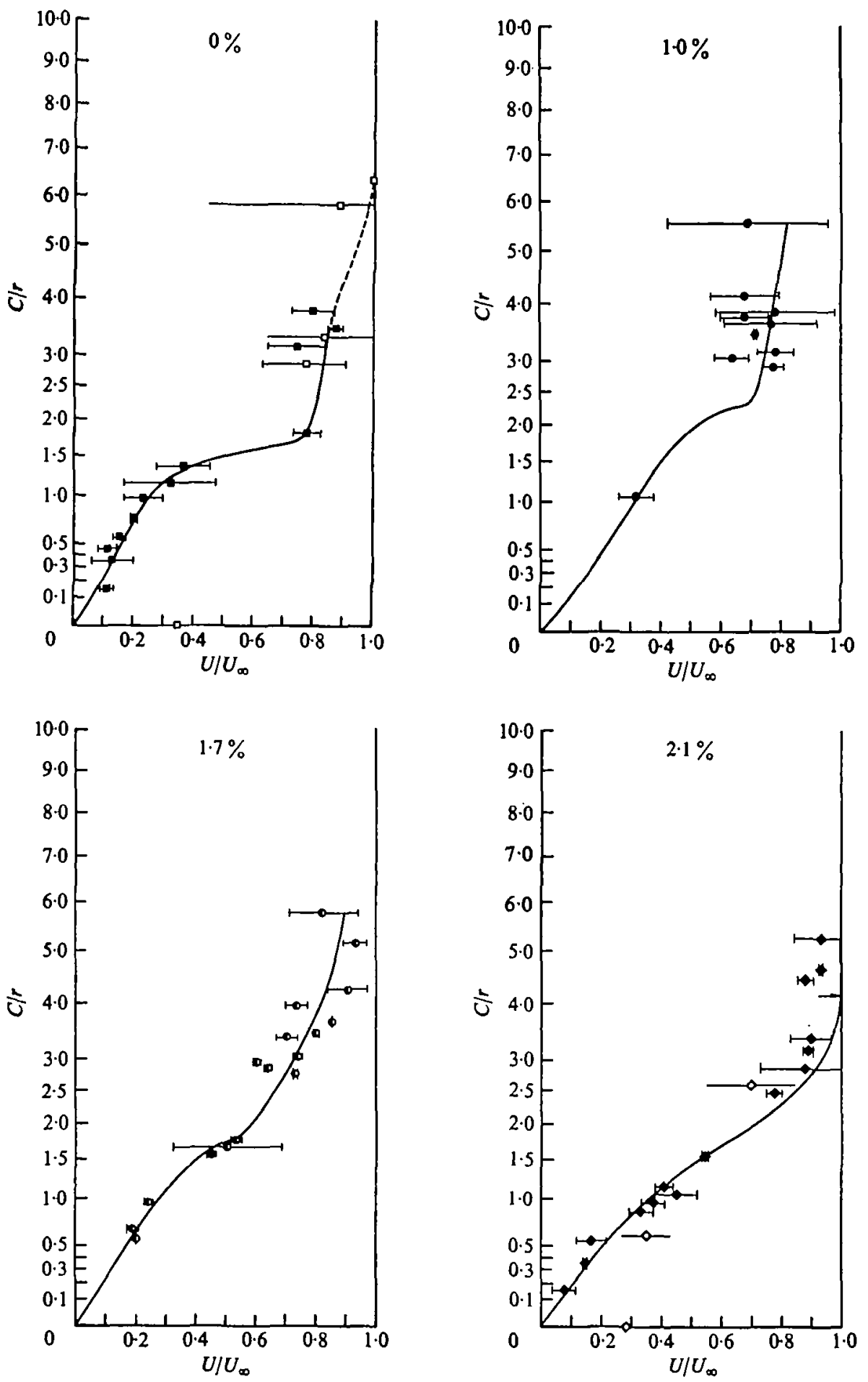

Fig. 6. For legend see p. 292. 

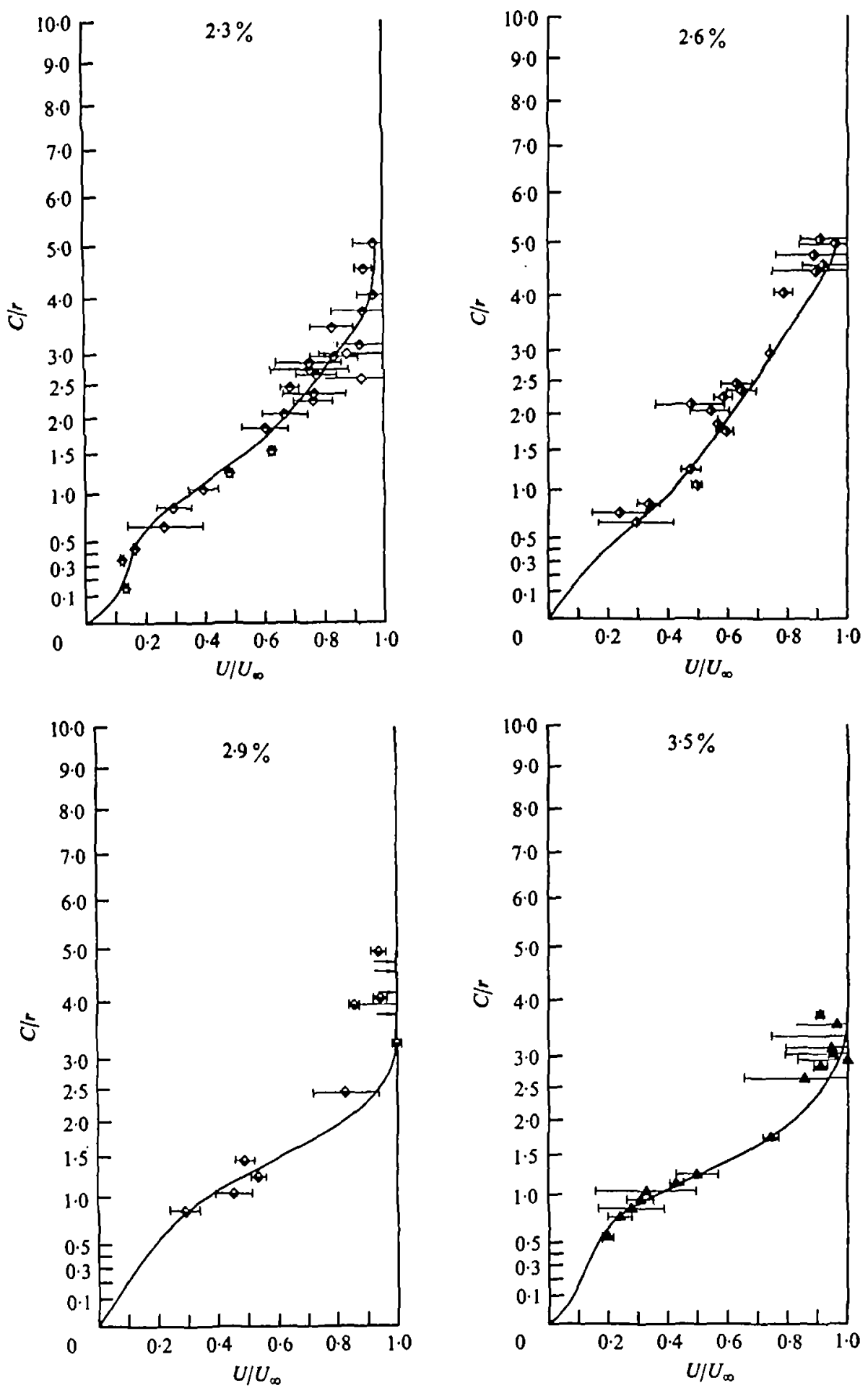

Fig. 6. For legend see p. 292. 

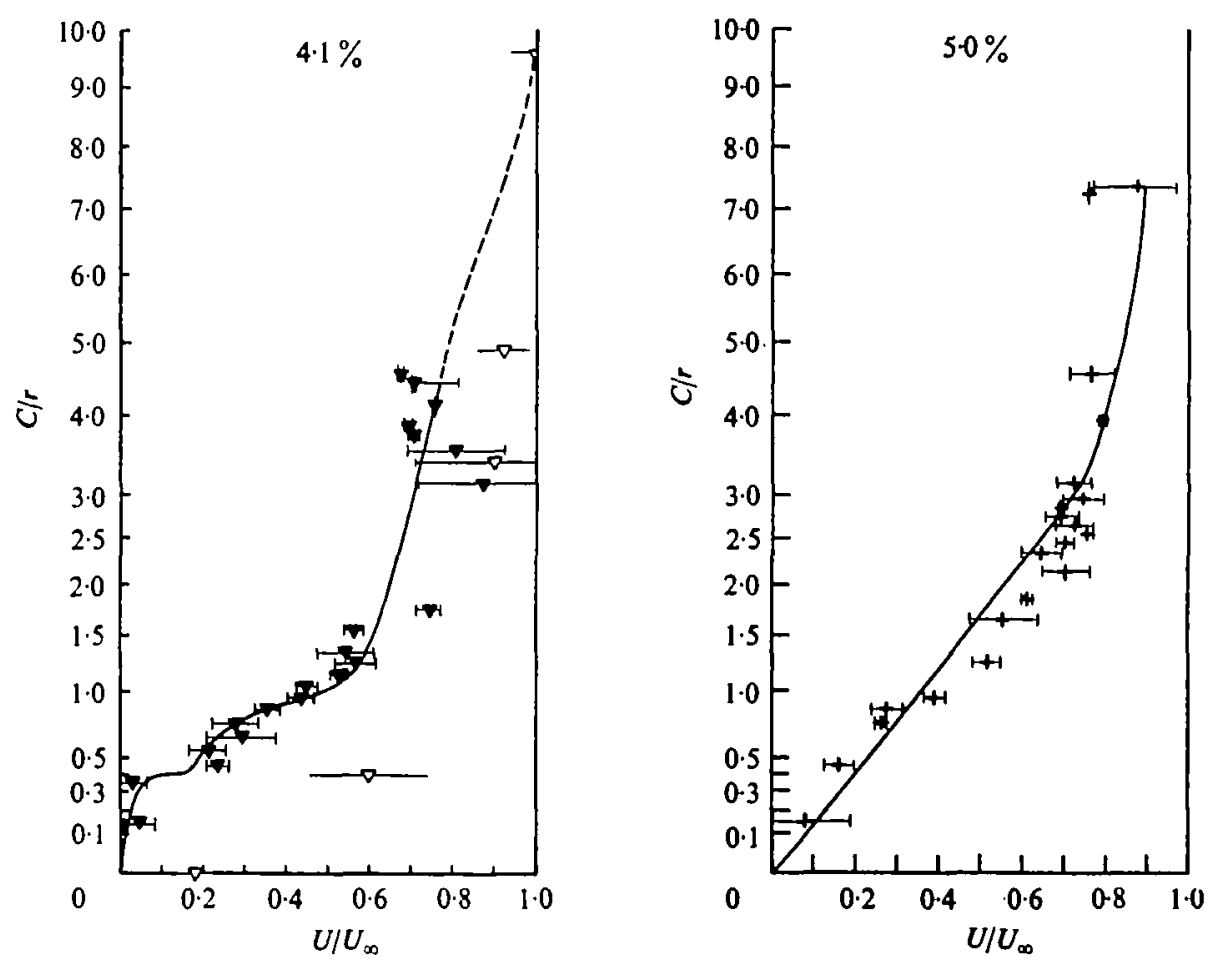

Fig. 6. Two-thirds power plots of data obtained from photographs of Tetrahymena swimming in tubes. Clearances and velocities were measured directly for each individual. Velocity values were then averaged according to the clearance range to which they belonged. The $C / r$ ranges utilized were $0-0.29$ (plotted at 0.15 ) $0.30-0.39,0.40-0.49,0.50-0.59, \ldots, 1 \cdot 10-1 \cdot 19$, etc. (plotted at $0.35,0.45,0.55, \ldots, I_{15}$, etc., respectively). The completely open symbols of the $0 \%, 2.1 \%, 2.3 \%$ and $4.1 \%$ graphs represent data from the timed experiments rearranged to fit the $C / r$ range scheme utilized here. Dashed lines are utilized where $C / r$ values from the timed experiments extend above photographic data.

and organism end effects are so much more important than lubrication per se, that the shape of the organism must be considered and its velocity more usefully expressed in body lengths per second $L / s$. Such a recalculation and graphing of the data has been performed along with a study of the relationship of body length to width in the growing organism. The resulting plots, although differing from Fig. 6 in the coordinates of a number of mean values, did not display any deviations that would invalidate any conclusions based upon Fig. 7 .

The regions of interest, then, where mucin-caused wall-drag reduction is considered, are the first and last clearance ranges and the tendencies described above may be evaluated by a statistical analysis. To simplify the analysis we chose a representative $C / r$ from each range; for the first $C / r=0.70$ and for the last $C / r=3.5^{\circ}$. Then after choosing an equal number of plot mean values (from Fig. 6) above and below the representative $C / r$ value, a regression line was determined utilizing the Caltech IBM subroutine REGRES and if the line had a slope $\neq 0$ then all $U / U_{\infty}$ observation values for $C / r \neq 0.70$ (or $3.5^{\circ}$ ) were normalized to their most probable value at $C / r=0.70$ 


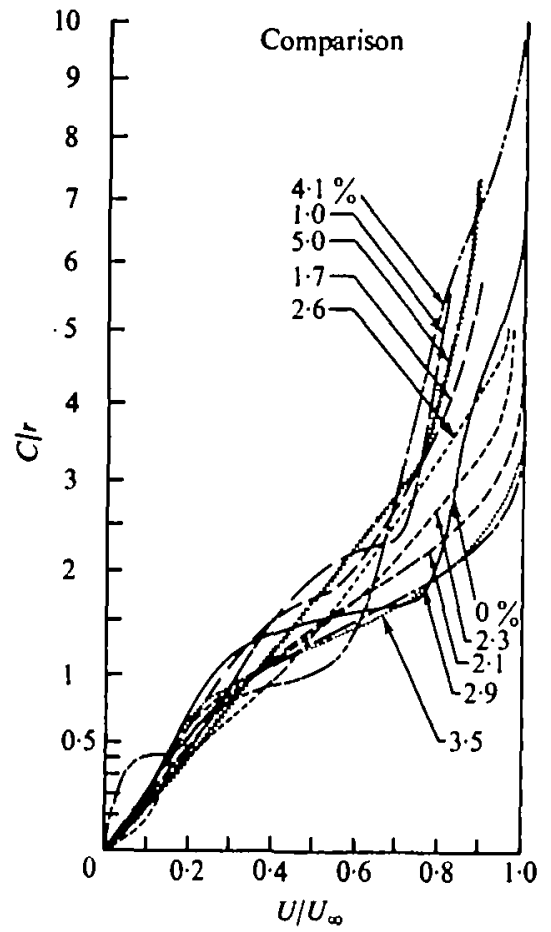

Fig. 7

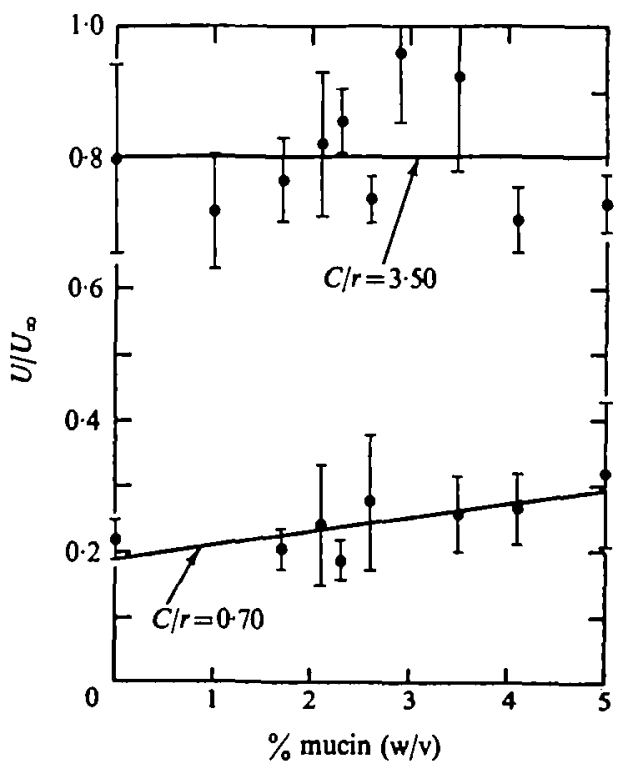

Fig. 8

Fig. 7. A combined plot of all the curves from Fig. 6 without error bars. The lubrication effect of mucin appears in the $C / r$ range $0.40-1.0$ and disappears by $C / r=1.5$. An optimized nonlubricative drag reduction occurs above $C / r=3.5$.

Fig. 8. Regression lines for wall-drag at two clearances as a function of mucin concentration. Each point represents scattered $U / U_{\infty}$ data which has been normalized to its value at the clearances given by a multivariate regression IBM subroutine REGRES. The regression line presented is generated by the same subroutine.

\section{Table I}

$\begin{array}{clccc}C l r & \begin{array}{c}\text { Mucin conc. } \\ \text { range }(\%)\end{array} & N & t_{0.005} & t_{\text {aux. }} \\ 0.70 & 1 \cdot 7-5.0 & 147 & 2.75 & 21 \cdot 01 \\ 3.50 & 1.7<M<4.1 & 241 & 2.75 & 22.50 \\ & M<2.1-3.5<M & 321 & 2.75 & 20.80\end{array}$

Student's $t$ test (Tippett, 1941) for the hypothesis that each of the presented populations of $U / U_{\infty}$ values is not a member of the control population. The $t_{0.00}$ level given signifies what value must be erceeded in order to achieve $99 \%$ confidence that the hypothesis has been verified. The $N$ values given are the sample sizes for each range. The value of $t$ is calculated from $t=\left(\bar{x}_{1}-\bar{x}_{\varepsilon}\right)\left[s_{t}\left(N_{0}^{-1}+N^{-1}\right)^{1}\right]^{-1}$, where $\bar{x}_{2}-\bar{x}_{2}$ is the absolute value of the difference of the means of the control and test samples, $s_{i}^{2}=\left(s_{a}^{2}+s_{N}^{2}\right)\left(N_{0}-1+N-1\right)^{-1}, s_{0}$ is the standard deviation of the control sample mean, $s_{y}$ the standard deviation of the test sample mean, $N_{\mathrm{c}}$ the size of the control sample.

(or 3.50 ). The means and standard deviations of the resulting sample sets could then be calculated as representative of the two clearances.

The subroutine REGRES was again applied to these two sets of data and the resulting regression lines are presented in Fig. 8. The non-zero slope of the $C / r=0.70$ plot shows that the lubrication effect is significant and tends to increase with mucin 


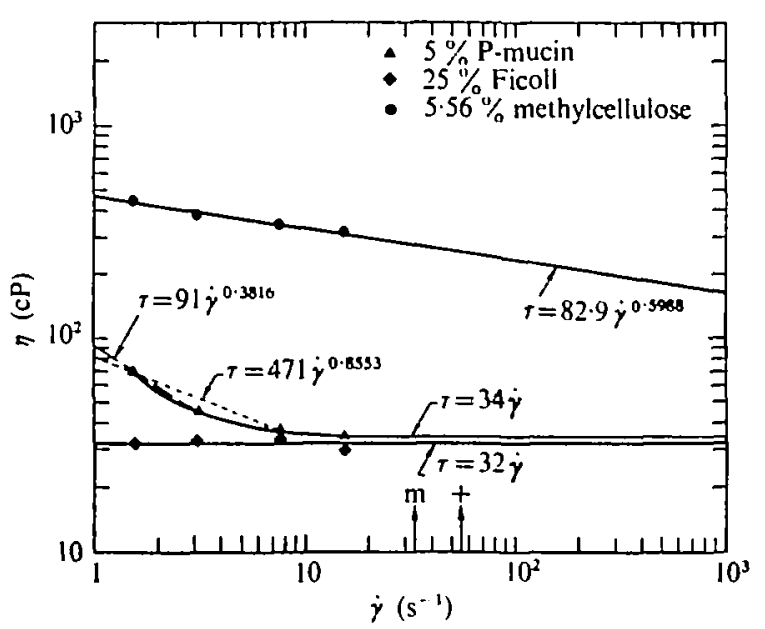

Fig. 9. Log-log shear-viscosity plots of Brookfield viscometer measurements of three types of polymer suspensions. Where clear tendencies are apparent, the plots are thick solid lines. Power law relationships calculated from $\eta=\tau / \dot{\gamma}$ are included near the appropriate curves. Estimates of possible power law curves for the non-linear portions of the mucin curve are indicated by dashed lines and the apparent Newtonian portion of the mucin curve is indicated by a thin solid line. Typical shear rates for cilia in normal medium $(+)$ and in methylcellulose $(\mathrm{m})$ are indicated by arrows arising from the abscissa. It is assumed that the tendencies interpreted from the given measurements do not change in the shear rate range covered by this graph.

concentration. A zero slope of the $C / r=3.5^{\circ}$ plot indicates no reduction of wall drag by mucin but this interpretation ignores the optimized character of the mucin effect around $C / r=3.5$ shown in Fig. 7 , which would be consistent with a net zero regression line slope. The significance of the wall drag reduction effect may be further evaluated by subjecting the data from Fig. 8 to Student's $t$ test; the results of which are listed in Table I. Two particularly noteworthy conclusions in which one canthave at least $99 \%$ confidence follow directly from the $t$ test. First, mucin is a lubricant for objects propelled by cilia near walls and the lubrication is concentration dependent up to at least $5 \%$. Secondly, wall drag on objects propelled by cilia outside the lubrication region is increased significantly by mucin concentrations outside the range $\mathrm{I} \cdot 7<M<4 \cdot \mathrm{I} \%$ and decreased significantly by concentrations inside this range; a pattern which is, by definition an optimized effect.

The power law estimate of $\eta_{0}$ was obtained from a $\log -\log$ plot of $\eta$ as a function of the shear rates $\dot{\gamma}$ utilized in viscometry. The viscometric values were then extrapolated to $\dot{\gamma}$ values associated with the ciliary tip as calculated from published measurements. The power law is usually stated as the shear stress $\tau=K \dot{\gamma}^{n}$, where $K$ is a constant related to viscosity and $n$ is a measure of how close a fluid is to being Newtonian (where $n=\mathrm{I}$ ). For such a power law fluid, the apparent $\eta$ at any fixed shear rate is related to the shear rate by $\eta=K \dot{\gamma}^{n-1}$. Accordingly, one can obtain the standard power law form by multiplying $\eta=K \dot{\gamma}^{n-1}$ by $\dot{\gamma} \dagger$. For Tetrahymena cilia in normal medium, we obtain a shear rate of $\dot{\gamma}=55^{.85} \mathrm{~s}^{-1}$, and for an externally measured $\eta$ of $50 \mathrm{cps}$ in methylcellulose $\dot{\gamma}=33.5^{-1}$ given a $7 \mu \mathrm{m}$ cilium tracing a projected arc of

$\dagger$ Here the viscosity at any point is taken to be $\eta=\tau / \dot{\gamma}$ (Han \& Barnett, 1973), which is the value a Newtonian fluid would have under the same conditions. 
$80^{\circ}$ and beating at about 20 and $12 \mathrm{~Hz}$ respectively as calculated from Preston (1972). (The projected arc lengths and frequencies of cilia in very narrow tubes are not known but are probably smaller than the values given.) In any case, the shear rates are probably still too great to render the net viscous relationships non-Newtonian. Shear stress relationships for the three polymers are presented in Fig. 9. Ficoll behaves as a Newtonian fluid at the shear rates utilized. Methylcellulose is non-Newtonian as expected, but remains simple enough to conform to a power law plot throughout the range of shear rates utilized, and the extrapolated plot is taken to be a straight line within the shear range represented on the graph. Mucin, in contrast, does not conform to a power law relationship until the shear rates are sufficient to render the suspension Newtonian. In any case, it is reasonable to suppose that prior to the transformation from non-Newtonian to Newtonian the fluid may exhibit power law kinematics. Accordingly, two extrapolations which approximate power laws for mucin are included in the figure: the first is obtained by extending the line connecting the lowest two $\dot{\gamma}$ measurements, and the second by extending the line joining the first and third $\dot{\gamma}$ measurements. While these extrapolations move away from the values associated with the cilia, they remain relevant because cilia do not move at a constant velocity and may experience non-Newtonian viscosity conditions during the slower portions of their beat cycle. Moreover, the viscometry measurements given here apply to the less viscous PGM. The more viscous BSM may extend its non-Newtonian influence to shear rates nearer $30 \mathrm{~s}^{-1}$.

\section{CONCLUSIONS AND DISCUSSION}

The most significant finding of these investigations is the significant lubrication behaviour of mucin at the concentrations investigated. The velocity profiles for these concentrations in the narrow clearance tubes are typical of lubrication profiles in that they show an approach to a $U / U_{\infty}$ of unity significantly closer to the propulsive surface than is the case for the control. All three polymers show some evidence of lubrication behaviour, but the data for mucin are more convincing because of the greater precision of the photographic observations.

The need for lubrication by a surface suspended in a fluid and moving near a wall is a result of the no-slip boundary condition at the wall. The no-slip boundary condition is in turn a consequence of the fact that all real walls are rough on a microscopic scale such that 'the energy lost through viscous dissipation as a fluid passes over and around these irregularities is sufficient to ensure that it is effectively brought to rest' (Richardson, 1973). For a viscous Newtonian fluid, the tendency for fluid layers to adhere to each other is relatively great and the no-slip condition extends its influence (which we term 'wall drag' or 'wall effect'), some distance, $d$, from the wall. The exact value of $d$ varies with the roughness of the wall, the forces generating the fluid flow, and the viscosity of the fluid. As an example, a tetrahymenid sedimenting in normal medium in a glass tube will still experience an $8 \%$ slowing in its descent velocity some 108 body radii (129r) away (Winet, 1973). By reducing its entrained fluid volume to a sphere of viscous influence $80 \mathrm{me} 6.5 \mathrm{r}$ in radius, the swimming ciliate (or affixed ciliated field at a slightly larger value) can come quite near an opposing wall with no impairment of its propulsion. As it approaches closer than $d$, however, 
the total body experiences increasing wall drag until, eventually, it can generate no propulsion at all.

In the presence of a Newtonian lubricant, absolute propulsion rates are usually decreased in the open fluid and are further reduced near the wall: they are never reduced to zero, as long as the cilia can beat, because the fluid layers can slide past one another well enough to keep the wall's no-slip condition from reaching the ciliated surface. The results obtained from Tetrahymena swimming in Ficoll (Fig. 5) illustrate this kind of lubrication, although not dramatically.

A non-Newtonian lubricant responding to increased shear or dynamic pressure may show a non-uniform behaviour pattern (the dynamic pressure and its ultimate contribution to propulsion becomes quite significant where $C / r$ is small such that $L / C \gg \mathrm{I}$, where $L$ is the body length or the metachronal wavelength $\lambda$ ). In the mucin case such non-uniform behaviour is exemplified in the variation of curve forms in the first clearance range. Recent results with long-chain polymer suspensions in high $R e$ flow may, however, provide some insight into the mechanism for the lubrication behaviour of mucin.

It has been known for some time that the presence of long-chain polymers in a fluid flowing through a pipe at high $R e$ will reduce considerably the frictional drag exerted by the fluid (the 'Toms phenomenon'). While the individual polymer molecules have been the major concern of most investigators, Cox, Dunlop \& North (1974) have now established that polymer molecule aggregates are far more capable of drag reduction than are individual molecules. These investigators conclude that drag reduction is optimal at some aggregate size which is a function of shear rate and polymer molecular weight. Their model is not explored further at the aggregate level, but when considered along with evidence for localization of the polymer drag reduction mechanism at the wall (Lee, Vaseleski \& Metzner, 1974), and the demonstration by Hand \& Williams (1973) that a layer of adsorbed-entangled polymer molecules at a liquid/solid interface is a major contributor to drag reduction, it can be used to construct an explanation for drag reduction by polymers at low $R e$ (normally such transfers of mechanisms are not valid because drag is due much less to viscosity at high $R e$ than it is at low $R e$ ).

By forming a layer over the wall, a long-chain polymer such as mucin commences the reduction of viscous drag by eliminating the direct influence of the no-slip condition at the wall on bodies moving in the bulk fluid. Such bodies now see a wall consisting of a polymer layer, and as long as the dispersing fluid remains between the layer and the suspended particles-plus-body system, fluid viscosity will dominate propulsion. With increase in concentration, however, mucin gelates (Marshall, 1972) and its disperse particles aggregate with increasing incidence in accordance with the sol-gel transformation model of Bobalek et al. (1964). Eventually, a system evolves in which a polymer-layered wall is in loose contact, with a polymer cluster or gel coacervate, which is in turn in loose contact with a ciliated surface. In the system of present concern, if the cilia can propel mucin gel coacervates with sufficient velocity to produce a shear-stress-generated solating layer between the gel cluster and the mucin-layered wall, a substantial increase in slippage or lubrication will result. The gels here are seen as sliding past one another in a manner analogous to that of Teflon sheets. From the data presented in Fig. 9 it is evident that if we can assume a ciliary beat frequency 
in mucin at least as great as $f$ in the more viscous methylcellulose, then ciliary shear iates are sufficient to transform mucin flow from a gel-like non-Newtonian state to a solated Newtonian state.

When the gel concentration is high enough linkages may be formed which strengthen its integrity such that the organism may become effectively 'entangled' in the resulting network. The existence of such networks have been postulated for cervical mucus (Chretien et al. 1973) and we have observed the formation of a gel core at a mucin concentration of $9.1 \%$ which not only tends to exclude the swimming ciliate but gradually pulls apart in the narrowest bore section of the tube leaving a clear fluid in which two polar clumps of mucin are suspended. A picture of one of these clumps is presented in Fig. I2. An attempt was made to obtain a velocity profile for the $9 \cdot 1 \%$ mucin system but the measurements were undependable because of the 'contraction' of the mucin core. Notwithstanding, this lack of direct quantitative evidence for an upper limit to the concentration of mucin in which cilia can generate propulsion, the existence of such a limit is a reasonable assumption. This assumption coupled with the control system which acts as a lower lubrication limit defines a rather broadly optimized lubrication effect whose peak would lie in the mucin concentration range $\circ \%<M<9.1 \%$. Given such optimized lubrication as a foundation one can postulate a mucin lubrication model whose verification must await an even finer analysis than the photographic technique utilized in this investigation, which is to say a technique which can generate simultaneous measurements of fluid flow near the tube wall and within the ciliary layer.

The model that emerges from these considerations presumes that optimized behaviour of mucin lubrication with increase in concentration is readily explained by an increase in gel cluster size until the clusters or resulting networks are too large to be moved. At low concentrations, disperse phase clusters are too small and the particles too sparse to alter significantly the dispersing fluid rheology. At very high concentrations, cluster size is so large, and in contact with so much wall mucin, that in effect a gel network is formed which presents too much surface for the cilia to propel; and. the beating organelles merely carve solated channels where they come in contact with the gel clusters.

Such a model is illustrated in Fig. Io. It predicts critical cluster sizes for each of the three lubrication conditions. Minimum lubrication occurs where the clusters are particles so small that they do not significantly interrupt a cilium-to-wall fluid continuum. Moreover, the particles may become entangled in the ciliary layer. This lubrication condition is illustrated in Fig. Ioa. Maximum lubrication occurs where the clusters are more coacervate-like, being too large to become entangled in the ciliary layer yet small enough to be propelled by the cilia over the wall mucin (Fig. Iob). A gradual decline from maximum lubrication occurs as the clusters continue to increase to large aggregates which can no longer be propelled over the wall mucin. The mechanism for clustering is not of itself important to the model; any binding force sufficient to keep the cluster from 'breaking up' under ciliary shear stress would be adequate.

To obtain some indication of the feasibility of such a model, a series of Nomarskiinterference contrast photomicrographs was taken of various mucin suspension concentrations utilizing a Zeiss WL microscope system. The suspensions were prepared 
(a)

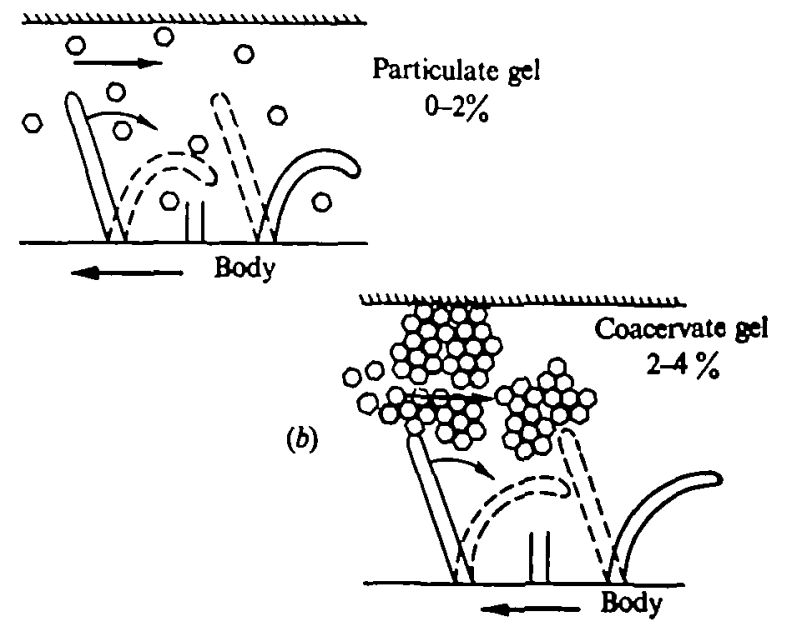

(c)

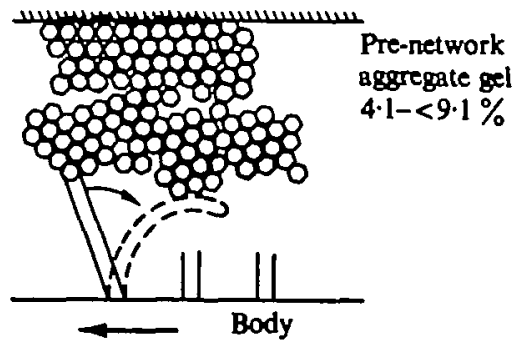

Fig. 10. A model for lubrication by mucin based upon the gelation theory of Bobalek et al. (1964). (a) At low concentrations mucin particles are too dispersed and sol-like to behave in a non-Newtonian fashion and they move freely in and out of the ciliary layer. (b) At optimal concentrations mucin forms clusters - called coacervates here - which are large enough to keep from getting entangled in the ciliary layer but small enough to be propelled over one another particularly when the more distal cluster is attached to the tube wall. The sliding of one cluater over another is facilitated by a shear-generated solation interface between the two clusters. Solation is indicated in the figure by the escaping single particles at the left of the interfacing clusters. (c) At high mucin concentrations the clusters are engaged in the building of a gel network. The large cluaters can still be propelled well enough to allow slippage in the clearance range where lubrication applies. However, their collisions in the open fluid with each other and the organism seriously affect the value of $\delta$. By the time a full network is formed lubrication disappears due to the absence of movable mucin clusters and $\delta$ is either quite large as the ciliated surface is in effect a component of the wall-fluid structure or drops sharply because the gel coagulates leaving the cell free to swim in a sol layer between the coagulated mass and the tube wall.

as described above, placed on a Vaseline-ringed slide and photographed at $20^{\circ} \mathrm{C}$. Sample results are presented in Fig. II. A Tetrahymena is visible at the left of the first panel and can be used for size comparison. Each mucin particle appears as a double image in the figure because of Airy disc overlap (this effect was verified with dark ground lighting under which the particles appeared as single images). The growth of cluster size between mucin concentrations of $x \cdot 5 \%$ and $2.25 \%$ does not appear to be dramatic but the incidence of particle formation has in fact doubled. The size of the particles with dark ground lighting is about $2 \mu \mathrm{m}$, which is too large to fall between cilia of Tetrahymena which are along the same meridian and about I $\mu \mathrm{m}$ apart, but they could fall between meridians which are about $3 \mu \mathrm{m}$ apart at their widest separation 


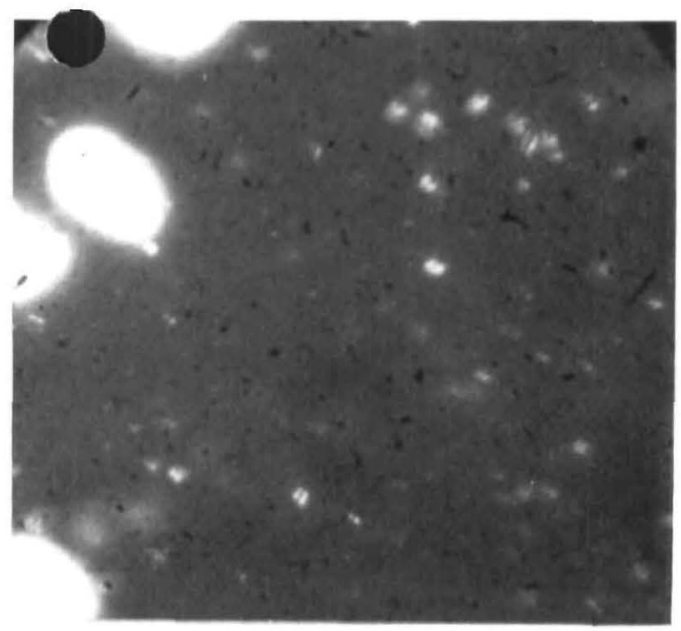

$1.50 \%$

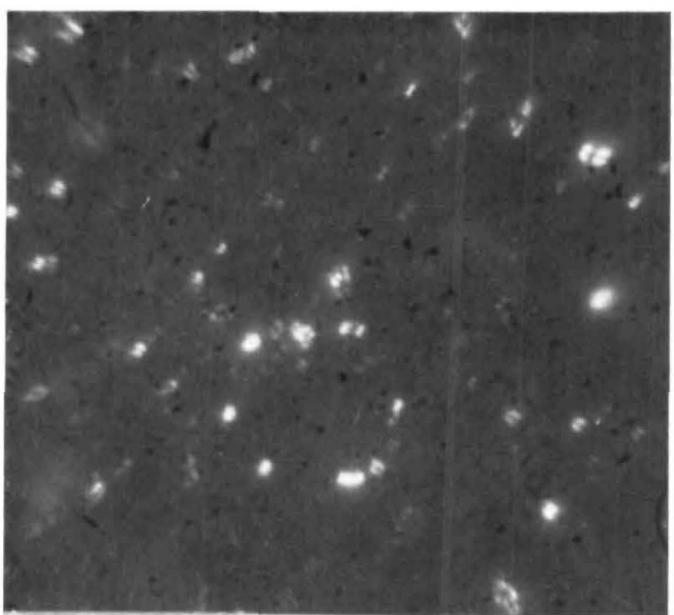

$2 \cdot 25 \%$

$10 \mu \mathrm{m}$

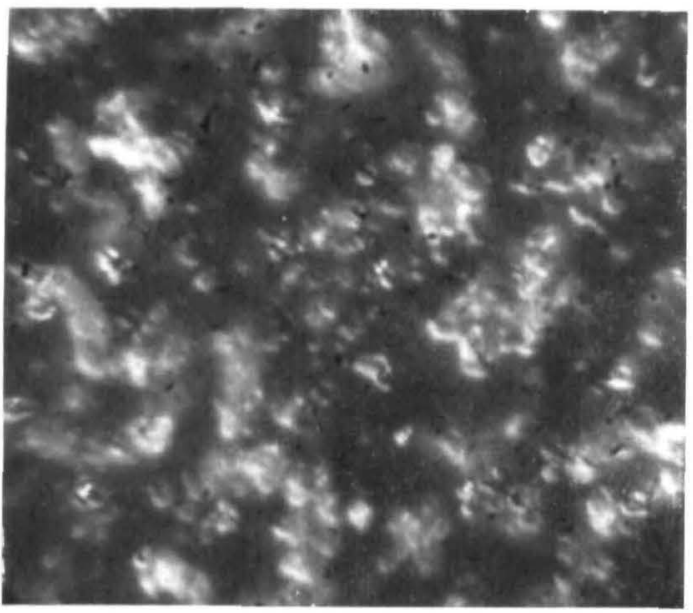

$4.50 \%$

Fig. 11. Photomicrographs of mucin suspensions in proteose-peptone Tetrahymena cultures. Three organisms can be seen at the left of the first panel. All percentages are in $w / v$. The average diameter of the $1.5 \%$ suspension clusters is about $2 \mu \mathrm{m}$, which is small enough to slip into the $3 \mu \mathrm{m}$ average meridian spacing of Tetrahymena. The $2.25 \%$ suspension clusters are about twice the diameters of the I'5\% suspension clusters. Given that the cilia of Tetralymena do not beat parallel with their meridians, it is evident that these cluaters would have difficulty penetrating the layer as predicted by the model (see Fig. 10) for the lubrication clearance range. The clusters in the $4.5 \%$ suspension are almost the size of the organism but can still be moved by the cilia. 


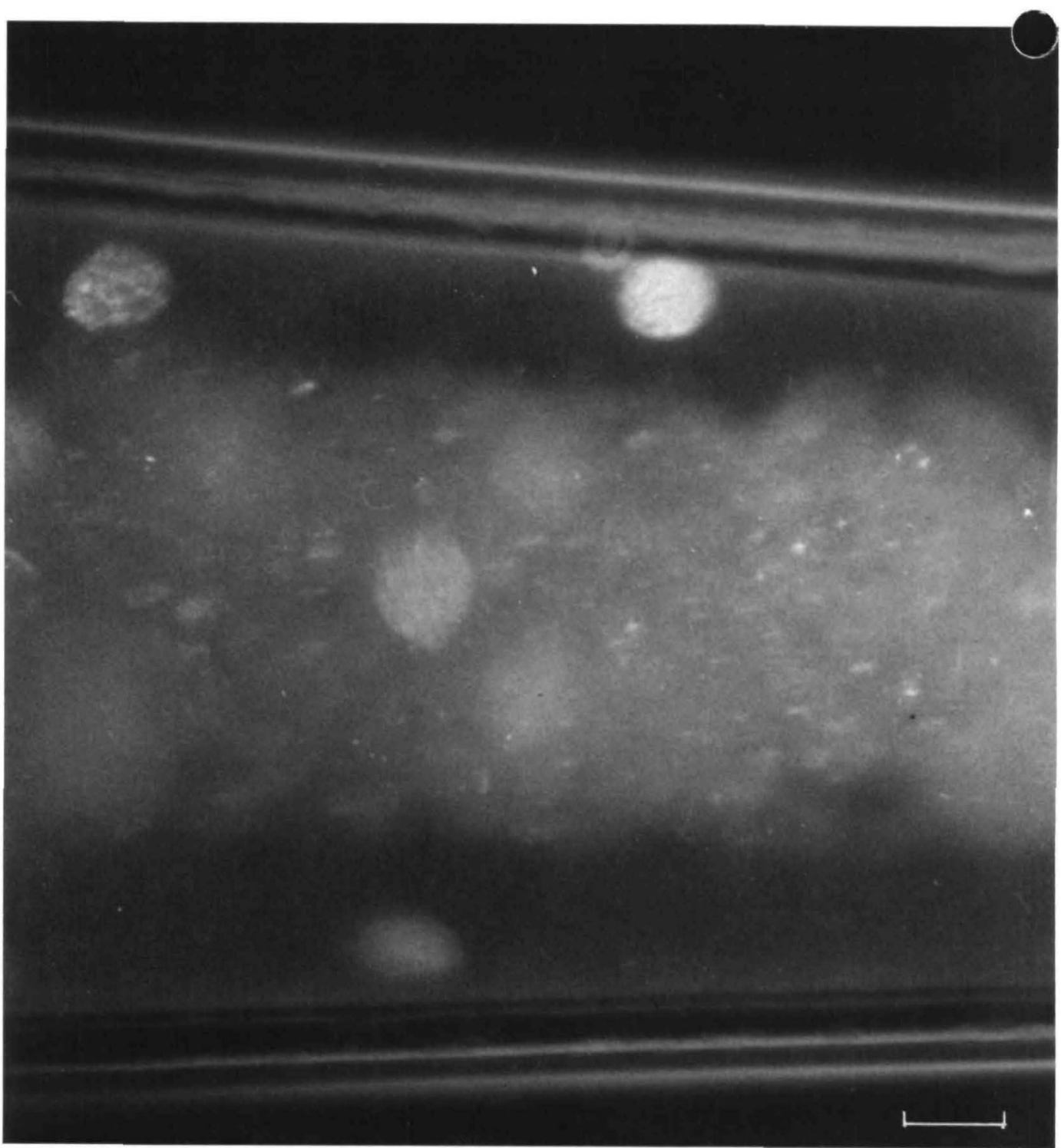

Fig. 12. Tetrahymena in a tube suspension of $9 \cdot 1 \%$ BSM. The gel network has formed sufficiently for the mucin to act as a continuous mass. Here the mass has contracted and exposed a sol or fluid region between itself and the glass wall. The organism tends to swim in the more fluid region making measurements of $U / U_{\infty}$ in $9.1 \%$ mucin difficult to obtain and meaningless with respect to lubrication nssessment. The scale line represents $30 \mu \mathrm{m}$.

\section{H. WINET}


Ythe separation falls to less than $\mathrm{I} \mu \mathrm{m}$ at the anterior and posterior ends, but these 'cannot approach the wall close enough to have a significant effect if the organism is swimming parallel to the wall). (Anatomical distances are measured from figures of Williams \& Scherbaum, 1959.) A cluster of two particles could still penetrate the ciliary layer if orientated appropriately, but non-linear clusters of 3 or more particles would experience great difficulty, especially if the ciliary beat enters the space between the meridians. The cilia of Tetrahymena do not beat parallel with their meridians (Preston, 1972) but an assessment of the maximum cluster size capable of penetration awaits the result of a more detailed microscopic analysis of ciliary propulsion in mucin. In any case, clusters $2 \mu \mathrm{m}$ or more wide will have difficulty penetrating the ciliary layer. The large clusters developed by suspension concentration levels of $4.5 \%$ or more are often as large as Tetrahymena, but not clearly too large to be moved. The enormous mass of gel formed at $9 \cdot 1 \%$ mucin which appears in Fig. 12 is certainly too large to be moved. Moreover, the fact that it forms a core cluster some distance from the wall disqualifies it as a lubricant. Accordingly, the growth of cluster size with concentration, indicated in Figs. I I and I2, does qualitatively support the proposed mucin lubrication model.

It may seem, given the simplicity of this model, that any similarity between it and the in vivo ciliated epithelium systems is minimal. On the contrary, recent scanning electron microscope studies by Van As \& Webster (1974) of the so-called 'mucous blanket' in pulmonary airways show that the blanket is in truth a suspension of mucus 'droplets, flakes and plaques'. While the lung epithelium cannot be considered a tube and the role of lubrication in the lungs is somewhat dubious, the characterization of the mucus suspension is our present concern; and the fact that mucus in the lungs is normally a $2-3 \%$ mucin suspension provides some basis for assuming a similarity between its structure and that of the mucin suspensions used in the present study. In addition, the SEM work of Chretien et al. (I973) noted above is consistent with the concept of gel network formation.

The results reported here suggest some simplifications and some complications for present physical analyses of tube-bounded ciliary propulsion of mucin suspensions as well as of objects carried in such suspensions. First the simplifications. It is quite probable that net fluid flow within the ciliary layer may be assumed to be Newtonian as long as shear rates exceed $\mathrm{I} \mathrm{s}^{-1}$, and while there is a reduction in absolute organism velocity in the mucin concentration range utilized (see Fig. 2), the lubrication effect enhances ciliary freedom of motion. Moreover, at the higher mucin concentrations where the gel clusters are too large to penetrate into the ciliary layer, there is even less opportunity for beat frequency reduction by increased bulk viscosity.

The clearance beyond which lubrication effects may be ignored may be estimated as $x \cdot 5 r$ (see Fig. 7) for the moving ciliated surface and probably no more than $3^{r}$ for the tethered surface. This simplification allows an analysis to be limited to 'unbounded' fluid mechanics where it can be shown that the clearances in the in vivo system being modelled are beyond these minima.

Unfortunately, the optimized behaviour of mucin systems in the part of the unbounded fluid mechanics clearance range $(>3.5 r)$ introduces a serious complication. If one calculates $\delta$, the effective depth of penetration of the vorticity generated by the beating cilia from $\lambda\left(\mathrm{I}+R e_{\omega}^{2}\right)^{-1}$ (Winet, I973) he will note that $\delta=\lambda$ because 
$R e_{\omega}$, the oscillatory Reynolds number, is $\ll$ I for the systems investigated herein Moreover, if we maintain the assumption that $S_{w} \simeq \delta$ (Winet, 1973) then, since the purely linear viscous effect of the mucin suspensions on $\delta$ are accounted for by the non-dimensionalization of $U$, we expect that $\delta$ would change for each mucin concentration only if $\lambda$ changed. The dramatic change in $\lambda$ required to account for the observed variation in $S_{v}$ values would contradict the observations of Machemer (I972), who found that a 50 -fold bulk $\eta$ increase resulted in a mere $35 \%$ increase in $\lambda$ for the ciliated Paramecium. The value of $\delta$ calculated for Tetrahymena utilizing the $\lambda$ measurements of Preston (1972) is $4.46 r$ (this value is indicated on the graphs of Figs. 3-5) a value for which even a $35 \%$ increase would not generate sufficiently large $\delta$ values to approach the $S_{w}$ values for $\mathrm{I} \cdot 0 \%, \mathrm{r} \cdot 7 \%, 4 . \mathrm{I} \%$ and $5 \%$ indicated in Fig. 7. Moreover, the states of the mucin suspensions at $1 \%$ and $5 \%$ are so different that a single mechanism explaining the $S_{w}$ pattern is unlikely. What is probably taking place in the lower concentration solutions is a non-Newtonian, i.e. non-linear purely hydrodynamic viscous effect which may be due to some hysteresis in the unsteady propagation of vorticity. In the higher concentration solutions one may have the rough equivalent of a porous medium in which large gel clusters are colliding with each other and the swimming organism. Here, of course, propulsive energy dissipation occurs by the transfer of momentum during collisions. There are other complications created by the formation of gel clusters. The interaction of the large clusters is often non-hydrodynamic. Non-uniform sol $\leftrightarrow$ gel conversions generated local viscosities which may deviate significantly from the bulk $\eta$. Such conversions also involve electrostatic interactions which may effect flow not only in the $>3.5 \mathrm{r}$ clearance range but in the lubrication range as well because the cilia carry a surface charge and the glass tube wall undoubtedly carries one, too.

Finally, the observed effects of methylcellulose and Ficoll have a significance peculiar to their applications. It has been suggested that mechanochemical experiments utilizing methylcellulose are of limited application because this substance is non-Newtonian and one cannot estimate the values of $\eta_{e}$. The results summarized in Fig. 9, however, indicate that the methylcellulose used here does obey the simple power law $\tau=$ $471 \dot{\gamma}^{0.8553}$ in the shear range of cilia and flagella and one can readily calculate $\eta_{c}$ if one can measure $\dot{\gamma}$. Ficoll, in contrast, behaves as a Newtonian fluid in the shear range measured directly. However, the Ficoll plots (Fig. 5) do not follow the control locus as they should for a purely Newtonian fluid so there must be some non-Newtonian effect. Moreover, these plots are virtually identical, i.e. independent of concentration, and it is difficult to formulate a model for such profile behaviour. In addition, a Ficoll concentration of $25 \%(\mathrm{w} / \mathrm{v})$ is required before $\eta$ is great enough to slow ciliary propulsion significantly, and for some investigations this ratio of solid-to-liquid may have too great an effect on water associations in the organism. Accordingly, the system being slowed will probably determine the optimum slowing agent.

This work was supported by National Science Foundation Grant $\mathrm{GK}_{3} \mathrm{II} \mathbf{I}_{\mathrm{IX}} \mathrm{X}$ and a contract from the Office of Naval Research, Nooor4-67-A-0094-0012. I would like to thank Dr Stanley A. Berger and Dr Charles J. Brokaw for critical reading of the manuscript and Dr Christopher Brennen for many useful conversations. 


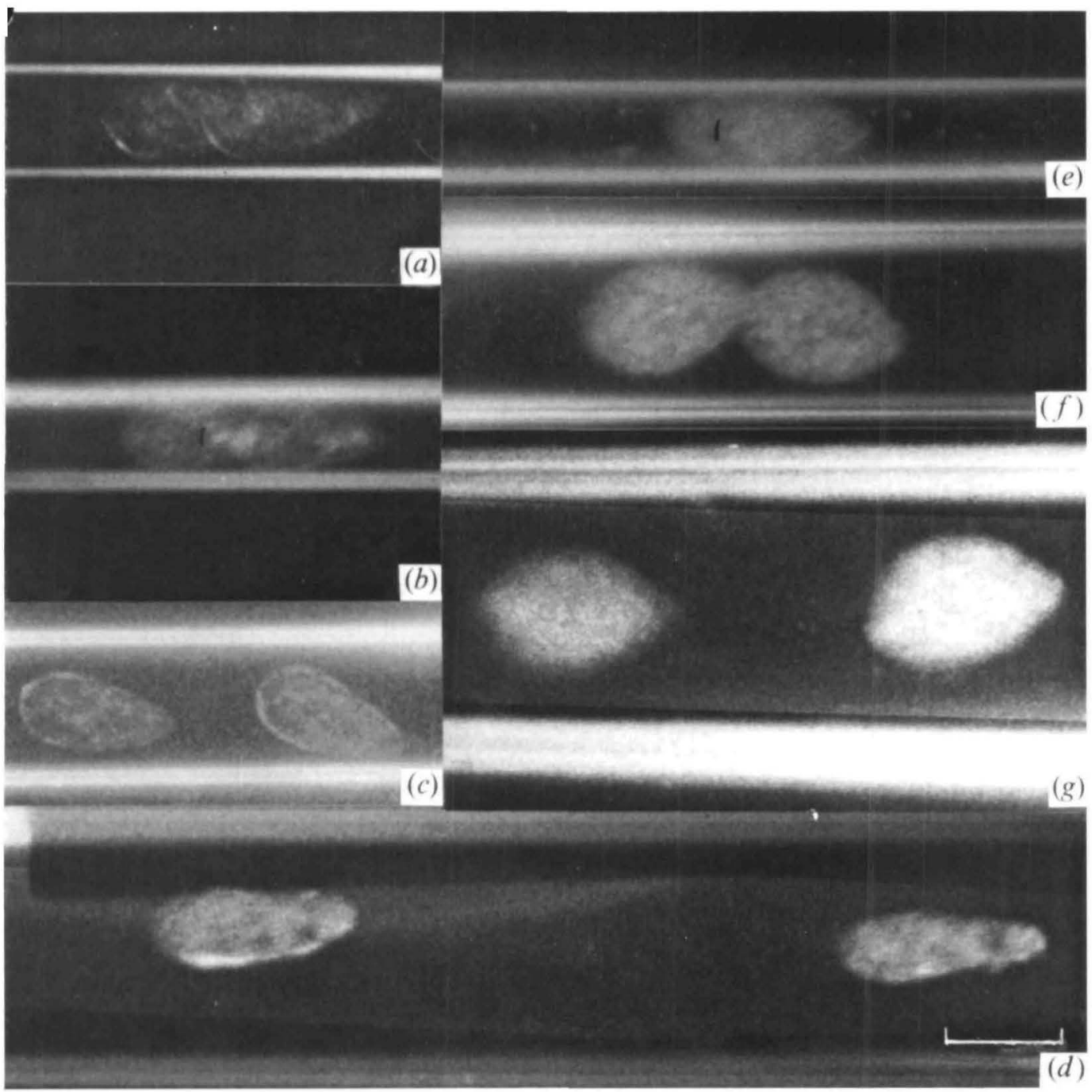

Fig. 13. Double pulse strobe imnges of Tetraliymena in tubes. Velocities are obtained from the progress of the posterior end which is partially outlined in black for clarity in some photographs. $(a-d) \mathrm{A} \circ \%$ mucin series taken at $2 \mathrm{pps}$. The clearances are in the ranges $(a) 0-0.20 r,(b) 0.40-0.49 r,(c) 1 \cdot 10-1 \cdot 15 r$ and $(d) 3 \cdot 10-3 \cdot 19 r$. (e-g) A $21 \%$ mucin series taken at. 1 pps with clearances $(e) 0-0 \cdot 29 r,(f) 1 \cdot 10-1 \cdot 19 r$ and $(g) 3 \cdot 10-3 \cdot 19 r$. The scale line represents $30 \mu \mathrm{m}$. 


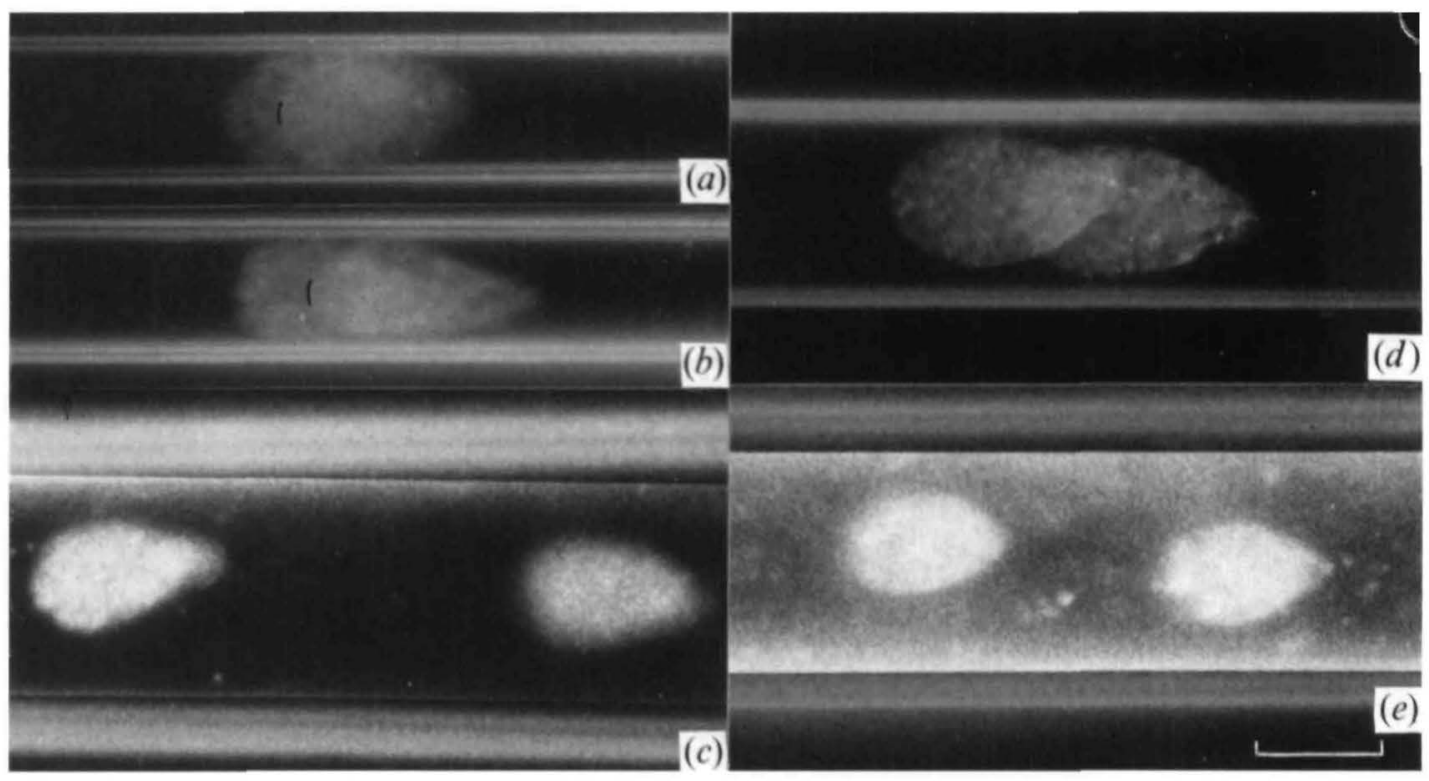

Fig. 14. Format details as for Fig. 13. Both series were taken at I pps. $(a-c)$ A $2.3 \%$ mucin series. Clearance ranges are $(a) 0-0.29 r,(b) 0.40-0.49 r$ and $(c) 3.10-3.19 r$. (d, e) A $3.5 \%$ mucin series with clearance ranges $(d) 1 \cdot 10-1 \cdot 19 r$ and $(e) 3 \cdot 10-3 \cdot 10 r$.

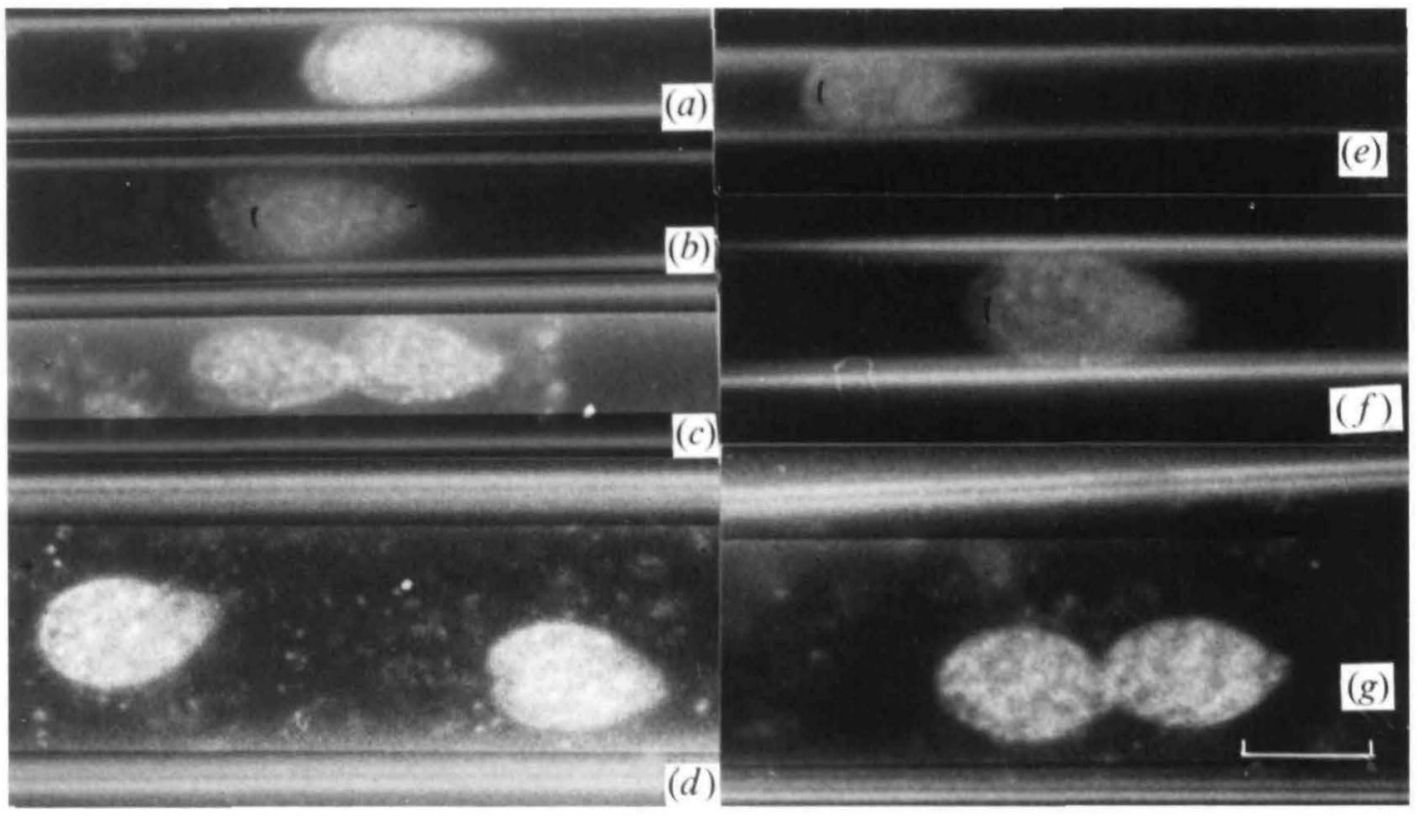

Fig. 15. Format details as for Fig. 13. Both series were taken at i pps. $(a-d)$ A $4.1 \%$ mucin series with clearance ranges $(a) 0-0.29 r,(b) 0.40-0.49 r,(c) 1 \cdot 10-1 \cdot 19 r$ and $(d) 3.1 c-3.10 r$. (e-g) A $5 \%$ mucin series with clearance ranges (e) $0-0.29 r,(f) 0.30-0.39 r$ and $(g) 3 \cdot 2 c-3 \cdot 29 r$. 


\section{Ciliary propulsion of objects in tubes}

APPENDIX : MEANING OF SYMBOLS

BSM bovine submaxillary mucin

C clearance between cell surface and tube wall

cP centipoise

$d$ radius of sphere of viscous influence as it relates to lubrication

$f \quad$ ciliary beat frequency

$K \quad$ power law constant

$n \quad$ power law exponent

$L \quad$ characteristic body length for lubrication calculations

$M \quad$ the concentration of mucin

PGM porcine gastric mucin

$P \chi^{2} \quad$ the probability that a sample is a member of the control population

Re Reynolds number (non-specific)

$R e_{\omega} \quad$ oscillatory Reynolds number

$S_{v} \quad$ distance from wall at which drag disappears

$r \quad$ average cilium length

$U \quad$ swim velocity in a bounded polymer suspension

$U_{0} \quad$ swim velocity in 'unbounded' normal culture medium

$U_{\infty} \quad$ swim velocity in 'unbounded' polymer suspension

$\dot{\gamma} \quad$ shear rate

$\delta \quad$ distance of significant diffusion of vorticity from the ciliate surface $\delta \simeq S_{v}$

$\eta \quad$ apparent viscosity of the bulk fluid

$\eta_{c} \quad$ local viscosity at the ciliary tips

$\lambda$ metachronal wavelength

$\tau \quad$ shear stress

$\theta \quad$ cilium long axis angle with body surface

\section{REFERENCES}

Barton, C. \& RAYnor, S. (1967). Analytic investigations of cilia induced mucous flow. Bull. math. Biophys. 29, 419-28.

Bevington, P. R. (1969). Data Reduction and Error Analysis for the Phyrical Sciences. New York: McGraw-Hill.

BLAKE, J. (1973a). Flow in tubules due to ciliary activity. Bull. math. Biol. 35, 513-23.

BLAKE, J. (1973b). Mucus flows. Math. Biosci. 17, 301-13.

BLAKE, J. (1973 c). A note on mucus shear rates. Resp. Physiol. 17, 394-99.

Bobalzk, E. G., Moore, E. R., Levy, S. S. \& LeE, C. C. (1964). Some implications of the gel point concept to the chemistry of alkyd resins. J. appl. Plym. Sci. 8, 625-57.

BRoKaw, C. J. (1966). Effects of increased viscosity on the movements of some invertebrate spermatozoa. 7. exp. Biol. 45, $113-39$.

Cheung, A. T. W. \& Winte, H. (1974). Flow velocity profile over a ciliated surface. In Sympasium on Sroimming and Flying in Nature: Paper Abstracts (ed. C. Brennen, C. J. Brokaw and T. Y. Wu). California Institute of Technology, Pasadena.

Chretien, F. C., Grenigon, C., David, G. \& Psychoyos, A. (1973). The ultrastructure of human cervical mucus under scanning electron microscopy. Fert. Steril. 24, 746-57.

Cox, L. R., Dunlop, E. H. \& NoRTH, A. M. (1974). The role of molecular aggregates in liquid drag reduction of polymers. Nature, Lond. 249, 243-5.

ELIFZRR, N. (1974). Viscoelastic properties of mucus. Biorheology II, 61-8.

Gibbons, R. A. \& GLoven, F. A. (1959). The physiochemical properties of two mucoids from bovine cervical mucin. Biochem. f. 73, 21 7-25.

HAN, C. D. \& BARNETT, B. (1973). Measurement of the rheological properties of biological fluids. In Rheology of Biological Systems (ed. H. L. Gabeinick and M. Litt). Springfield: Charles C. Thomas. 
HaNd, J. H. \& Williams, M. C. (I973). The adsorbed-entangled layer in drag reduction. Chem. Eng Sci. $28,63-8$.

LARDNER, T. J. \& SHACK, W. J. (1972). Cilia transport. Bull. math. Biopliys. 34, 325-35.

LeE, W. K., VASELESEI, R. C. \& MeTZNER, A. B. (1974). Turbulent drag reduction in polymer solutions containing suspended fibers. A.I.Ch.E.Yl 20, 128-33.

MACHEMER, H. (1972). Ciliary activity and the origin of metachrony in Paramecium; effects of increased viscosity. F. $\exp$. Biol. 57, 239-60.

Marshall, R. D. (1972). Glycoproteins. A. Rev. Biochem. 4r, 673-702.

MiLLER, C. E. (1969). Streamlines, streaklines, and particle path lines associated with a mechanically induced flow homomorphic with the mammalian mucociliary system. Biorheology 6, 127-35.

Pharmacia Fine Chemicals, Inc. (1968). Technical Bulletin for Ficoll.

Preston, J. T. (1972). Determination of a continuous helical ciliary beat in Tetrahymena pyriformis and the cytotic effect of serum complement from normal and cystic fibrotic sera on the organism. Doctoral thesis, University of California, Los Angeles.

Richardson, S. (1973). On the no-slip boundary condition. F. Fluid Mech. 59, 707-19.

Ross, S. M. (I97I). A wavy wall analytic model of muco-ciliary pumping. Ph.D. thesis, Johns Hopkins University.

TIPPETT, L. H. C. (1941). The Methods of Statistics. London: Williams and Norgate.

Tsuiki, S., Hashimoto, Y. \& Pigman, W. (1961). Comparison of procedures for isolation of bovine submaxillary mucin. 9. biol. Chem. 236, $2172-8$.

VAN As, A. \& WeBster, L. (1974). The morphology of mucus in mammalian pulmonary airways. Environ. Res. 7, I-I 2.

WaLters, K. (1973). New concepts in theoretical and experimental rheology. In The Rheology of Lubricants (ed. T. C. Davenport). New York: Wiley.

Williams, N. E. \& SchrRbaum, O. H. (1959). Morphogenetic events in normal and synchronously dividing Tetrahymena. 9. exp. Morphol. 7, 241-56.

WINET, H. (1973). Wall-drag on free-moving ciliated microorganisms. Y. exp. Biol. 59, 753-66.

Winet, H. \& JoNes, A. R. (r975). Mucocysts in the Heterotrich ciliate Spirostomum. F. Protozool. 22, 293-6. 